\title{
ENERGY EFFICIENT NODE RANK-BASED ROUTING ALGORITHM IN MOBILE AD-HOC NETWORKS
}

\author{
D. Kothandaraman ${ }^{1 *}$ and C. Chellappan ${ }^{2}$ \\ ${ }^{1 *}$ Department of Computer Science and Engineering, S R Engineering College, \\ Warangal, TS, India. \\ ${ }^{2}$ Department of Computer Science and Engineering, CEG, Anna University, \\ Chennai, TN, India.
}

\begin{abstract}
Mobile Ad-hoc Network (MANET) is an emerging technology, infrastructure less with self-organizing, selfhealing, multi-hop wireless routing networks in real time. In such networks, many routing problems arise due to complexity in the network mobility which results from difficulty in achieving energy efficient routing in the field of MANET. Due to the dynamic nature and the limited battery energy of the mobile nodes, the communication links between intermediate relay nodes may fail frequently, thus affecting the routing performance of the network and also the availability of the nodes. Though existing protocols are not concentrating about communication links and battery energy, node links are very important factor for improving quality of routing protocols because Node Rank helps us to determine whether the node is within transmission range or out of transmission range through considering residual energy of the node during the routing process. This paper proposes a novel Energy Efficient Node Rank-based Routing (EENRR) algorithm which includes certain performance metrics such as control overhead and residual energy in order to improve the Packet Delivery Ratio (PDR), and Network Life Time (NLT) from its originally observed routing performance obtained through other existing protocols. Simulation results show that, when the number of nodes increases from 10 to 100 nodes, EENRR algorithm increases the average residual energy by $31.08 \%$ and $21.26 \%$ over the existing Dynamic Source Routing (DSR) and Energy Efficient Delay Time Routing (EEDTR) protocols, respectively. Similarly it increases the PDR by $45.38 \%$ and $28.3 \%$ over the existing DSR and EEDTR protocols respectively.
\end{abstract}

\section{KEYWORDS}

Energy efficiency; Link stability; Node rank; Packet delivery ratio; Residual energy; Threshold energy

\section{INTRODUCTION}

A Mobile Ad Hoc Network (MANET) is an autonomous collection of mobile nodes which forms a dynamic wireless network. The administration of such a network is decentralized; each node acts both as host and router which forwards packets for nodes that are not within transmission range of each other [1]. A MANET provides a practical way to build a decentralized communication network rapidly in areas where there are no existing infrastructure or where temporary connectivity is needed (i.e.) situations such as emergency situations, disaster relief scenarios, military applications, etc. The topology of a Mobile Ad-hoc Network is very dynamic, which makes the design of routing protocols much more challenging than that for a wired network [2]-[4]. In general, routes between nodes in an ad hoc network may include multiple hops and hence it is appropriate to call such networks as multi-hop wireless ad hoc networks. The nodes in the MANET are battery operated. The failure of any node's operation can greatly impede the performance of the network and even can affect the basic availability of the network i.e., routing. The 
movement pattern, location, direction of movement, pause distribution, speeds and acceleration change over time of the mobile nodes can be described by their mobility models.

The various applications of MANET are search and rescue, automated battlefields, emergency relief scenarios, law enforcement, public meeting, data network, device network, virtual classroom, disaster recovery, sensor networks, and other security sensitive computing environment. The main characteristics of MANETs are a dynamic topology, node mobility (provides a large number of degree of freedom), Distributed operation, Multi hop routing, Autonomous terminal and self-organizing capability [5]-[6].

Ad-hoc wireless networks inherit the traditional problems of wireless communications such as bandwidth optimization, power control and transmission quality enhancement. Additionally, their mobility, multi-hop nature and lack of a fixed infrastructure create a number of complexities and design constraints that are new to mobile ad hoc networks. Some of the major MANET research issues are routing, power/energy constraint, limited bandwidth, dynamic topology, packet losses due to transmission errors, routing overhead, mobility-induced route changes and security threats [7]-[10]. To solve these issues various researchers chose different perspective as discussed in section 1.1.

\section{1. RELATED WORK}

To establish a communication route from a source node to a destination node, a sufficient number of intermediate nodes are required [11]-[12]. The Destination Sequenced Distance Vector (DSDV) routing protocol shows that wireless communication can be established without a base station and/or a fixed wire network. In DSDV, each node keeps track of other nodes in its neighborhood in an information table and records the desired information. When a source node initiates a routing path to the destination node, it selects a routing path based on the data stored in the information table. Additionally, an algorithm that allows the source node needs to choose the shortest routing path to the destination node. Another table driven approach is Wireless Routing Protocol (WRP) which lowers the memory and bandwidth. Hence, requires less storage for recording and operates only on-demand information.

Dynamic Source Routing (DSR) is an exemplary another class of on-demand algorithms, specifically designed to identify a routing path without the need of an information table [13]-[15]. The main drawback of DSR protocol is the generation of redundant route replies. These RREP travels through the path in which RREQ's bearing same Ids were received. This causes not only congestion but also wastage of battery power. Modified DSR (MDSR) has been designed to reduce control overhead by reducing the number of routing reply packets and a fixed header size for the data packets which acts itself as one of the energy efficient routing protocol [8], [16].

A source node can broadcast the routing path requirement message without obtaining the information from other nodes in the neighborhood first. To establish the routing path the routing request (RREQ) should reach the destination node. In order to find a more stable shortest routing path with a lower block probability, [17] Signal Stability Routing (SSR) protocol has been proposed, [18] which determines an intermediate node based on signal stability. Only when the signal stability of the node is within a transmission range the node will be selected as an intermediate node along with a routing path. Also Ad-hoc On-demand Distance Vector (AODV) routing protocol [19]-[20] scans and chooses the intermediate nodes by considering the on-demand distance-vector, thus lowering the overflow of routing 
path requirement. When an algorithm attempts to sift through potential routing paths for a shortest path, the same node which has been repeatedly utilized becomes highly resourceexhausted and may become more crowded over. Therefore, how to distribute traffic among various routing paths based on more parameters becomes an important issue.

Additionally, maintaining an established routing path is also an important issue. Depending on the dynamical state of the network, the established routing path may be blocked. If there is only one routing path from a source node to the destination node, the communication is deemed inflexible. Therefore, numerous Multi-path Routing protocols have been proposed where only the traffic on one routing path is examined,[17] and the traffic load is also not diverted into multiple routes. Also, Multiple Source Routing (MSR) was proposed to distribute the traffic using a weighted heuristic-based strategy [21]. The routing algorithm employs Round Trip Time (RTT) as the criterion. Though RTT is not the only parameter affecting the traffic load, it cannot reflect the real routing state. Therefore, additional parameters such as link stability and load balance have been considered.

MTPR (Minimum Total Transmission Power Routing) sets up the route that uses the lowest transmission power among possible routes. This can be applied in any environment where transmission power adjustment is available. Because the required transmission power is proportional to the $\mathrm{n}^{\text {th }}$ power of the distance between nodes, this scheme prefers shorter links and has the tendency to select the route with more hops. However, MTPR has some problems. It turns out that the adaptation of transmitting power that can bring a new hidden terminal problem which results in more collision and more energy consumption due to retransmission. And, MTPR has a similar problem like min-hop routing in that it makes no efforts to use energy evenly among nodes [22].

CMMBCR (Conditional Max-Min Battery Capacity Routing) tries to balance both the total transmission power consumption and the individual node power consumption. This algorithm operates in two modes according to the residual battery power. If there are nodes that have more battery power than threshold power, it applies MTPR to the nodes. Otherwise, it mimics MMBCR. When battery power is plentiful, it minimizes the total energy consumption like MTPR, and in the other case it considers the nodes with lower energy like MMBCR. The performance of CMMBCR is heavily influenced by the threshold value. In a case if threshold value is 0, then it is identical to MTPR [22]. If the threshold value grows by infinity, then it is transformed into MMBCR. However, it only depends on the threshold energy level only and it does not allocate energy utilization evenly throughout all nodes. It chooses a route whose bottleneck residual energy larger than a certain threshold. If there is more than one route satisfying this condition, then it selects the one with minimum total transmission power. Node Disjoint Multipath Routing Considering Link and Node Stability (NDMLNR) mainly aimed to find the multiple nodes disjoint routes from source to a given destination [23]. Also, it keeps track of the routing bandwidth which can be further used by the source to select the optimal routes. This NDMLNR uses the factors Link Expiration Time (LET) and Drain Rate (DR) to find the Link Stability. Higher the value of Link Stability Degree (LSD), the higher is the stability of the link and the greater is the duration of its existence. Thus, a route having all the links with $\mathrm{LSD}>\mathrm{LSD}_{\text {thr }}$ is the most feasible.

Energy Efficient Delay Time Routing (EEDTR) protocol differs from the existing methods [24], [25]. Here the neighbor node introduces a delay in forwarding the RREQ packet which is inversely proportional to its remaining energy level. Based on this protocol, the maximum remaining energy node forwards the RREQ packet first with a smaller delay and the minimum energy remaining node forwards the RREQ packet latter by a longer delay. 
Hence the destination node receives the first RREQ packet forwarded by the maximum energy remaining intermediate nodes. As soon as the destination receives the first RREQ packet, it transmits a RREP towards the source. The new modifications allow this node to remain alive for a longer time. This introduces a delay in the network which increases the end to end delay and reduces packet delivery ratio [26]. This has to be considered as a problem to be overcome. Though so many research articles have addressed the above routing issues, still these areas require more research to solve these issues in MANET. Hence, this research paper focuses on energy efficient routing paths based on Node Rank, with an aim to achieve high PDR and network life time by choosing energy based less traffic route. This paper also addresses the solution to some of these above issues like routing, packet losses and power / energy management by proposing a novel Energy Efficient Node Rank-based Routing (EENRR) algorithm.

\section{EnERgy EFFicient NODE RANK-BASEd ROUTING (EENRR) Algorithm}

Energy Efficient Node Rank-based Routing (EENRR) algorithm is proposed to increase Network Life Time (NLT) and Packet Delivery Ratio (PDR). In this algorithm, NLT of the nodes in the network is increased by choosing energy efficient path and PDR is increased by choosing minimal traffic root from source to destination nodes [27]. This improvement in NLT and PDR is achieved using Node Rank (NR) technique in EENRR algorithm [28]. Node Rank is evaluated based on the number of links connected to the node and the total number of nodes in the network. The DSR protocol is enhanced by modifying the route discovery but the route maintenance is essentially the same. Route selection is performed by choosing energy efficient path and less traffic routes by the source node using NR. Major part of the proposed EENRR algorithm concentrates in predicting the Node Rank which is described below. For an example figure 1 represents the sample for routing architecture, which acts as a base for evaluating the node rank

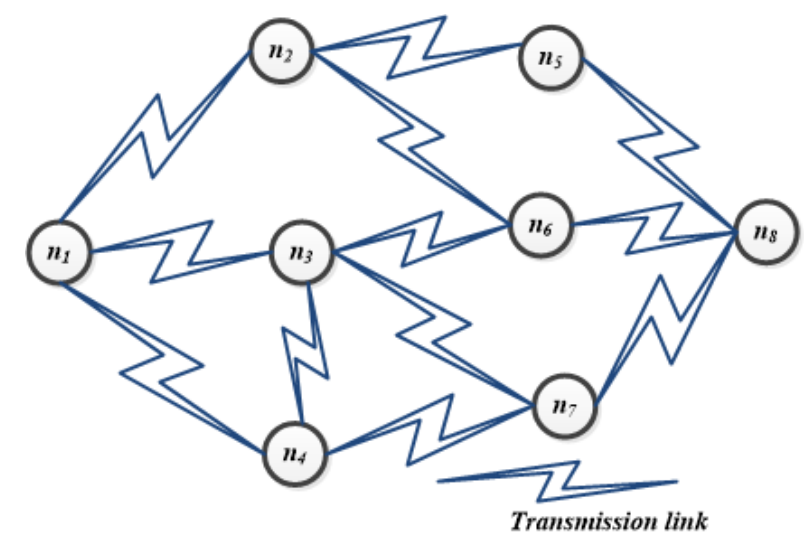

Figure 1.Sample routing architecture

\subsection{Node Rank (NR) evaluations}

Node Rank is a link analysis algorithm and it assigns a numerical weight to each node of a linked set of nodes, with the purpose of measuring its relative importance within the set. This numerical weight assigned to any given node $\mathrm{i}$ is referred to as the NR and it is denoted by $N R\left(n_{i}\right)$. The NR of a node is defined recursively, depending on its previous NR value and number of links connected to the node. A node that is linked to many nodes will have low NR value. High NR value with fewer neighbor links will increase the traffic and 
less NR will minimize the traffic. The proposed EENRR algorithm will select less NR routing path.

The following section assumes a probability distribution between 0 and 1 for Node Rank. Assume a small universe of eight nodes: $n_{1}, n_{2}, n_{3}, n_{4}, n_{5}, n_{6}, n_{7}, n_{8}$. Node Rank is initialized to the same value for all nodes which is computed as in equation 1 . Hence the initial value for each node is 0.125 . The node $\boldsymbol{n}_{\mathbf{2}}$ has link to nodes $\boldsymbol{n}_{\mathbf{1}}, \boldsymbol{n}_{\mathbf{5}}, \boldsymbol{n}_{\mathbf{6}}$, node $\boldsymbol{n}_{\mathbf{3}}$ had a link to node $n_{1}, n_{4}, n_{6}, n_{7}$ and node $\boldsymbol{n}_{\mathbf{4}}$ has links to node $n_{1}, n_{3}, n_{7}$.

\section{Iteration 1: Node Rank evaluation at time $t=0$}

At $t=0$, an initial probability distribution is assumed, usually

$$
\operatorname{INR}\left(n_{i}\right)=\frac{1}{N}
$$

Where INR $\left(n_{i}\right)$ is the Initial Node Rank value of node $n_{i}$. For the given routing example as shown in figure $2, \mathrm{~N}$ represents the total number of nodes which is 8 . Hence INR $\left(\mathrm{n}_{\mathrm{i}}\right)$ value set to all nodes as $1 / 8$ i.e., 0.125 .

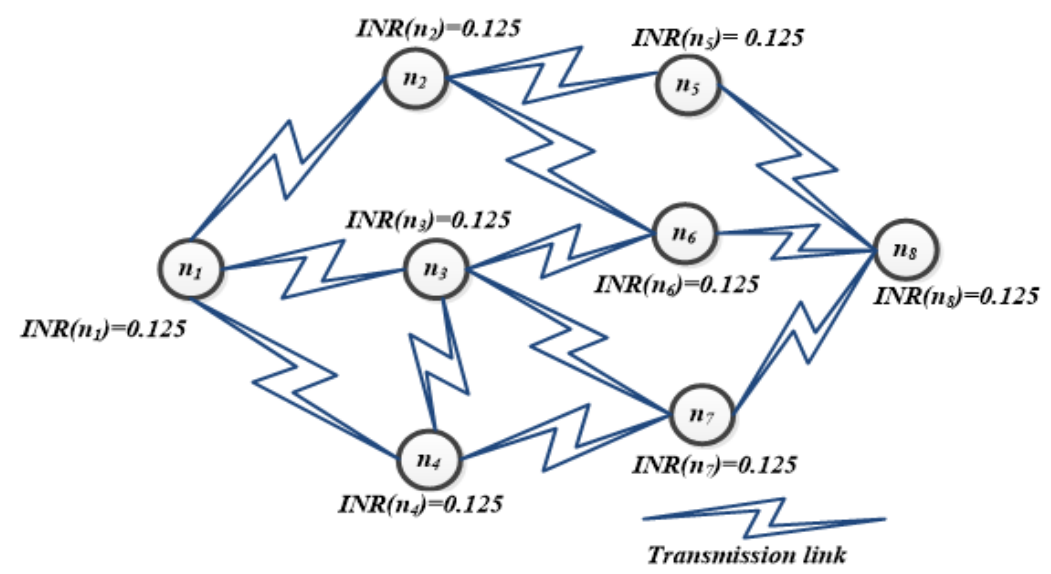

Figure 2. Network using initial node rank value

\section{Iteration 2: Node Rank evaluation at time $t=t+1$}

In the general case, the Node Rank value for any node $\mathbf{u}$ can be communicated as:

$$
N R(u)=\sum_{v \in\left(B_{u}\right)} \frac{N R(v)}{L(v)}
$$

The Node Rank for a node $\mathrm{u}$ is dependent on the Node Rank value for each node $\mathrm{V}$ contained in the set $\mathrm{B}_{\mathrm{u}}$ the set containing all nodes linking to node $\mathrm{u}$ divide by the number $\mathrm{L}(\mathrm{V})$ of links from node $\mathrm{V}$.

When considering damping factor(d) which can be thought of as the probability of nodes following the links and could regard $(1-d)$ as the Node Rank distribution from non-directly linked nodes. The generalised Node Rank evaluation is represented as follows

$$
N R\left(n_{i}\right)=\frac{1-d}{N}+d \sum_{n_{j} \varepsilon m\left(n_{i}\right)} \frac{N R\left(n_{j}\right)}{L\left(n_{j}\right)}
$$


Where $\mathrm{d}$ is a damping factor that is usually set to $0.85, n_{1}, n_{2, \ldots}, n_{N}$ are the nodes under consideration, $m\left(n_{i}\right)$ is the set of nodes that link to $n_{i}, L\left(n_{j}\right)$ is the number of outbound links on node $n_{i}$ and $N$ is the total number of nodes.

In iteration 2 follows equation 3, Node Rank is computed using its own previous Node Rank value and the number of outbound links. For example, the source node $n_{1}$ is linked to its neighbor nodes $n_{2}, n_{3}, n_{4}$, as shown in figure 2 , each link would transfer initial value 0.125 and its Node Rank is evaluated using equation 1.

$$
N R\left(n_{1}\right)=\frac{N R\left(n_{2}\right)}{L\left(n_{2}\right)}+\frac{N R\left(n_{3}\right)}{L\left(n_{3}\right)}+\frac{N R\left(n_{4}\right)}{L\left(n_{4}\right)}
$$

Where $\mathrm{L}\left(\mathrm{n}_{2}\right), \mathrm{L}\left(\mathrm{n}_{3}\right)$ and $\mathrm{L}\left(\mathrm{n}_{4}\right)$ is the number of links for node $\mathrm{n}_{2}, \mathrm{n}_{3}$ and $\mathrm{n}_{4}$ respectively. In the example network shown in figure 2 , it is observed that $L\left(n_{2}\right)=3$. Similarly $L\left(n_{3}\right)=4$, $L\left(n_{4}\right)=3$ and then substitutes in equation 4 as follows:

$$
N R\left(n_{1}\right)=\frac{N R\left(n_{2}\right)}{3}+\frac{N R\left(n_{3}\right)}{4}+\frac{N R\left(n_{4}\right)}{3}
$$

The NR value $N R\left(n_{2}\right)=\operatorname{INR}\left(n_{2}\right), N R\left(n_{3}\right)=\operatorname{INR}\left(n_{3}\right) \operatorname{and} N R\left(n_{4}\right)=\operatorname{INR}\left(n_{4}\right)$ and link values of related neighbor node are substituted in equation 4 which generates the NR value for node $\mathrm{n} 1$.

$$
N R\left(n_{1}\right)=\frac{1-0.85}{8}+0.85\left[\frac{0.125}{3}+\frac{0.125}{4}+\frac{0.125}{3}\right]
$$

$$
N R\left(n_{1}\right)=0.11575
$$

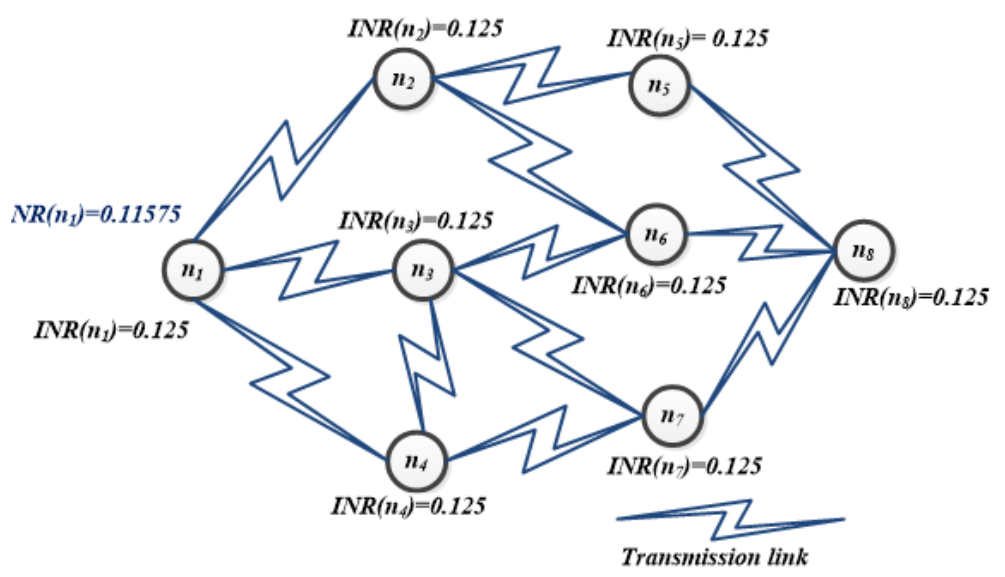

Figure 3. Node Rank updated network after first iteration

\section{Iteration 3: Node Rank evaluation at time $t=t+2$}

Similarly, at time $\mathrm{t}=\mathrm{t}+2$, Node Ranks for the nodes $\mathrm{n} 2, \mathrm{n} 3$ and $\mathrm{n} 4$ are evaluated using the following equations 3 ,

$$
\begin{aligned}
& N R\left(n_{2}\right)=\frac{1-d}{N}+d\left[\frac{N R\left(n_{1}\right)}{L\left(n_{1}\right)}+\frac{N R\left(n_{5}\right)}{L\left(n_{5}\right)}+\frac{N R\left(n_{6}\right)}{L\left(n_{6}\right)}\right] \\
& N R\left(n_{3}\right)=\frac{1-d}{N}+d\left[\frac{N R\left(n_{1}\right)}{L\left(n_{1}\right)}+\frac{N R\left(n_{6}\right)}{L\left(n_{6}\right)}+\frac{N R\left(n_{4}\right)}{L\left(n_{4}\right)}+\frac{N R\left(n_{7}\right)}{L\left(n_{7}\right)}\right] \\
& N R\left(n_{4}\right)=\frac{1-d}{N}+d\left[\frac{N R\left(n_{1}\right)}{L\left(n_{1}\right)}+\frac{N R\left(n_{3}\right)}{L\left(n_{3}\right)}+\frac{N R\left(n_{7}\right)}{L\left(n_{7}\right)}\right]
\end{aligned}
$$




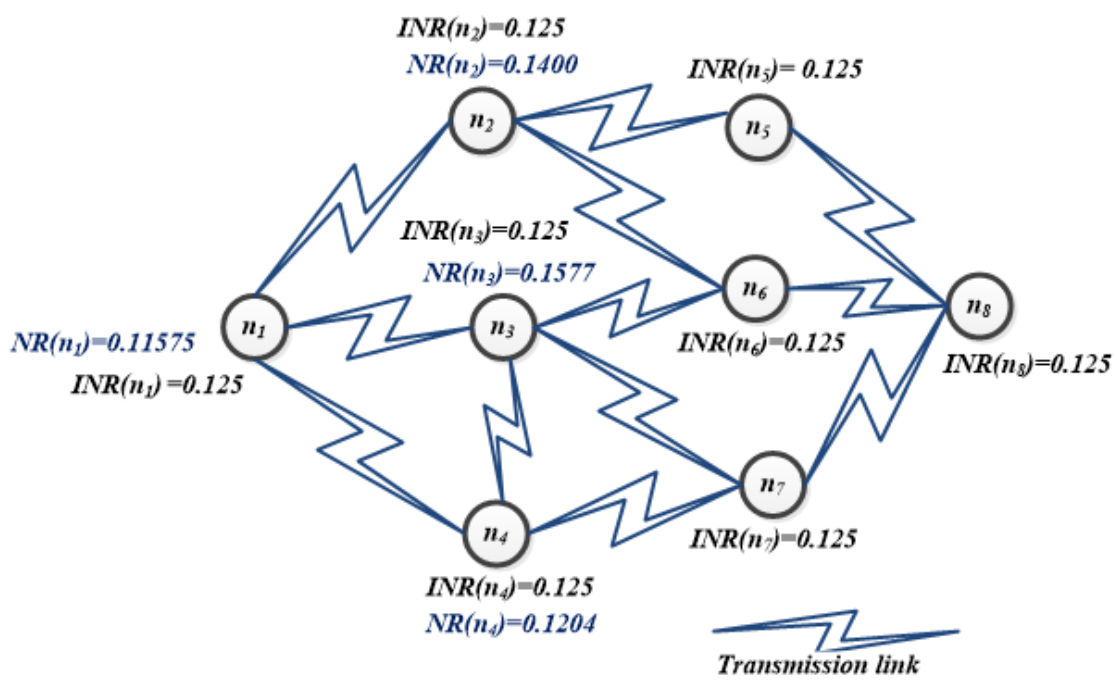

Figure 4. Node Rank updated network after second iteration

\section{Iteration 4: Node Rank evaluation at time $t=t+3$}

At time $\mathrm{t}=\mathrm{t}+3$, Node Ranks for the nodes $\mathrm{n}_{5}, \mathrm{n}_{6}$ and $\mathrm{n}_{7}$ are evaluated using the following equations 3 ,

$$
\begin{aligned}
& N R\left(n_{5}\right)=\frac{1-d}{N}+d\left[\frac{N R\left(n_{2}\right)}{L\left(n_{2}\right)}+\frac{N R\left(n_{8}\right)}{L\left(n_{8}\right)}\right] \\
& N R\left(n_{6}\right)=\frac{1-d}{N}+d\left[\frac{N R\left(n_{3}\right)}{L\left(n_{3}\right)}+\frac{N R\left(n_{2}\right)}{L\left(n_{2}\right)}+\frac{N R\left(n_{8}\right)}{L\left(n_{8}\right)}\right] \\
& N R\left(n_{7}\right)=\frac{1-d}{N}+d\left[\frac{N R\left(n_{3}\right)}{L\left(n_{3}\right)}+\frac{N R\left(n_{4}\right)}{L\left(n_{4}\right)}+\frac{N R\left(n_{8}\right)}{L\left(n_{8}\right)}\right]
\end{aligned}
$$

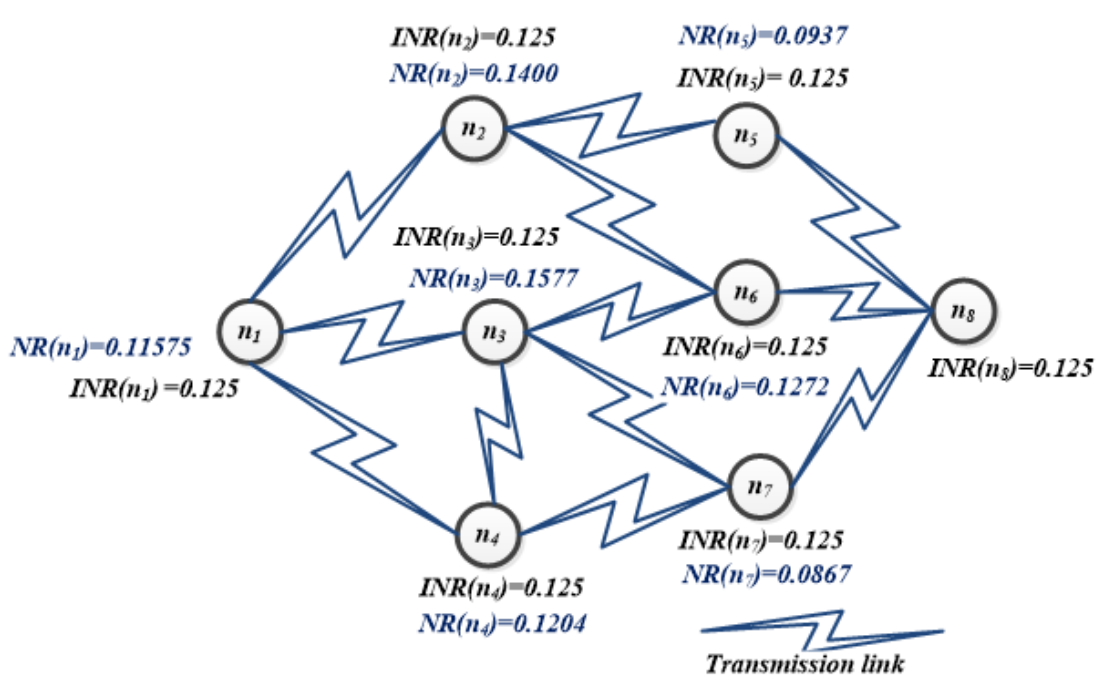

Figure 5. Node Rank updated network after final iteration

\subsection{Energy Efficient Node Rank-based Route Discovery Procedure}

The energy efficient route discovery is initiated by forwarding Route Request (RREQ) packets to its neighbor nodes based on its Node Rank value and Residual Energy $\left(E_{r}\right)$. Node Ranks are evaluated only for the neighbor nodes which may have a chance to forward the 
Route Request (RREQ) packet. Before forwarding RREQ to its neighbors source node computes Node Rank values for the related neighbors and then it compares their $E_{r}$ and Node Rank values. The RREQ is forwarded to the neighbors which has high residual energy than the threshold energy $\left(\mathrm{E}_{\mathrm{th}}\right)$ with less Node Rank value. Otherwise, the RREQ is simply rejected. Assume the threshold energy $\mathrm{E}_{\mathrm{th}}$ to be $30 \mathrm{mWhr}$. Though $E_{r} \geq E_{t h}$ but Node Rank is higher, then the RREQ is not forwarded to that neighbor. If $E_{r} \geq E_{t h}$ and Node Rank is lesser then RREQ is forwarded to the corresponding neighbor node. In case, if $E_{r}$ is less than the $\mathrm{E}_{\mathrm{th}}$, though the Node Rank value is less, the RREQ is rejected. Instead of flooding RREQ, this proposed EENRR algorithm, forwards the RREQ only to its neighbor satisfying the above criteria. Thus the control packets are reduced considerably, minimizing the traffic in the network, which in turn increases the NLT and PDR over the other existing protocols [29].

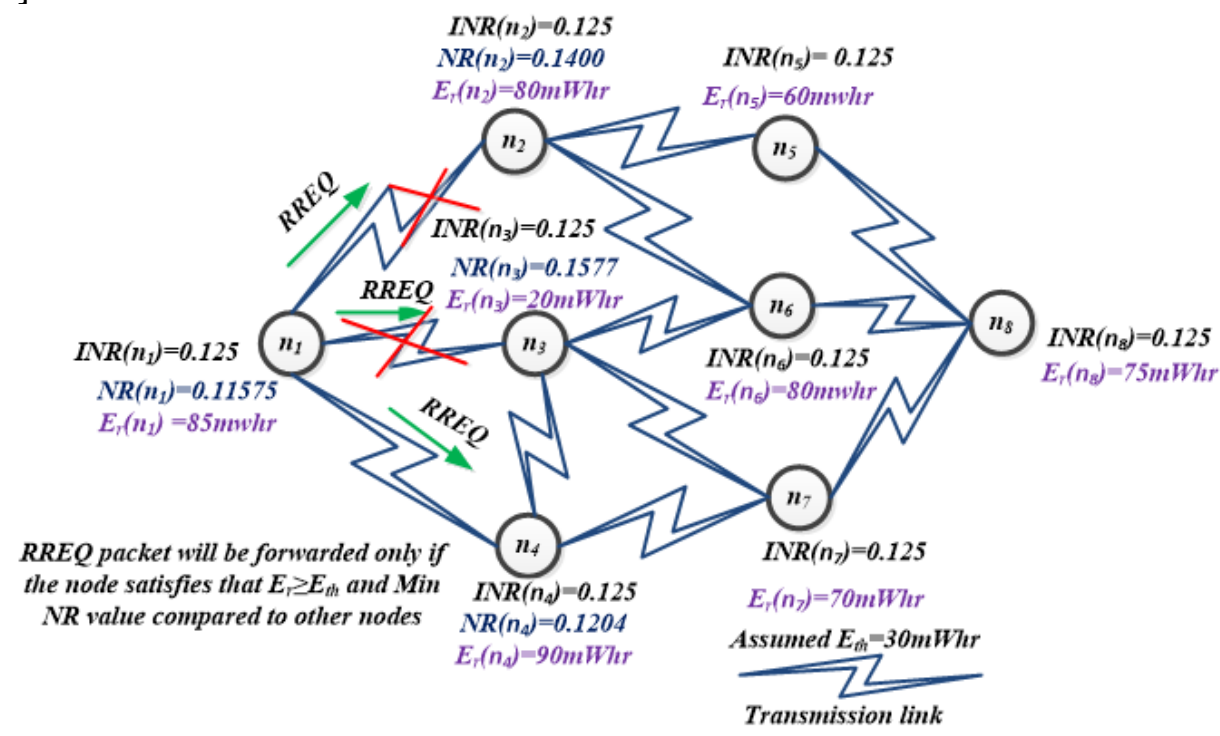

Figure 6. Source node forwarding RREQ packetto neighbor nodes

Route discovery is initiated by forwarding RREQ packets to its neighbor as shown in figure 6. Assume $\mathrm{n}_{1}$ as source node and $\mathrm{n}_{8}$ as destination node. Now $\mathrm{n}_{1}$ tries to forward RREQ to its neighbor nodes such as $\mathrm{n}_{2}, \mathrm{n}_{3}$ and $\mathrm{n}_{4}$. Before forwarding RREQ packets to its neighbors $\mathrm{n}_{2}, \mathrm{n}_{3}$ and $\mathrm{n}_{4}$, source node $\mathrm{n}_{1}$ compares the Node Rank values and $\mathrm{E}_{\mathrm{r}}$ values of them. As per the proposed EENRR algorithm, neighbor node $n_{3}$ has $E_{r}$ value less than $E_{t h}$, RREQ is not forwarded to node $n_{3}$. But, neighbor nodes $n_{2}$ and $n_{4}$ are having $E_{r}$ values higher than the $E_{t h}$ value. Hence, the RREQ will be forwarded to either of these two nodes which are having lesser Node Rank value. From the example network, node $\mathrm{n}_{4}$ is not only having higher $\mathrm{E}_{\mathrm{th}}$ but also having lesser Node Rank value compared to node $\mathrm{n}_{2}$. Hence, RREQ packet is forwarded to neighbor node $\mathrm{n}_{4}$ by source node $\mathrm{n}_{1}$. Similarly node $\mathrm{n}_{4}$ will forward RREQ packet to its neighbor node $\mathrm{n}_{7}$ which forwards the RREQ packet to the destination node $\mathrm{n}_{8}$. When the destination node receives the RREQ packet, it immediately sends back the Route Reply (RREP) packet to source node using the route information present in the RREQ packet as shown in figure 7 and its flow chart representation is shown in figure 8 . 


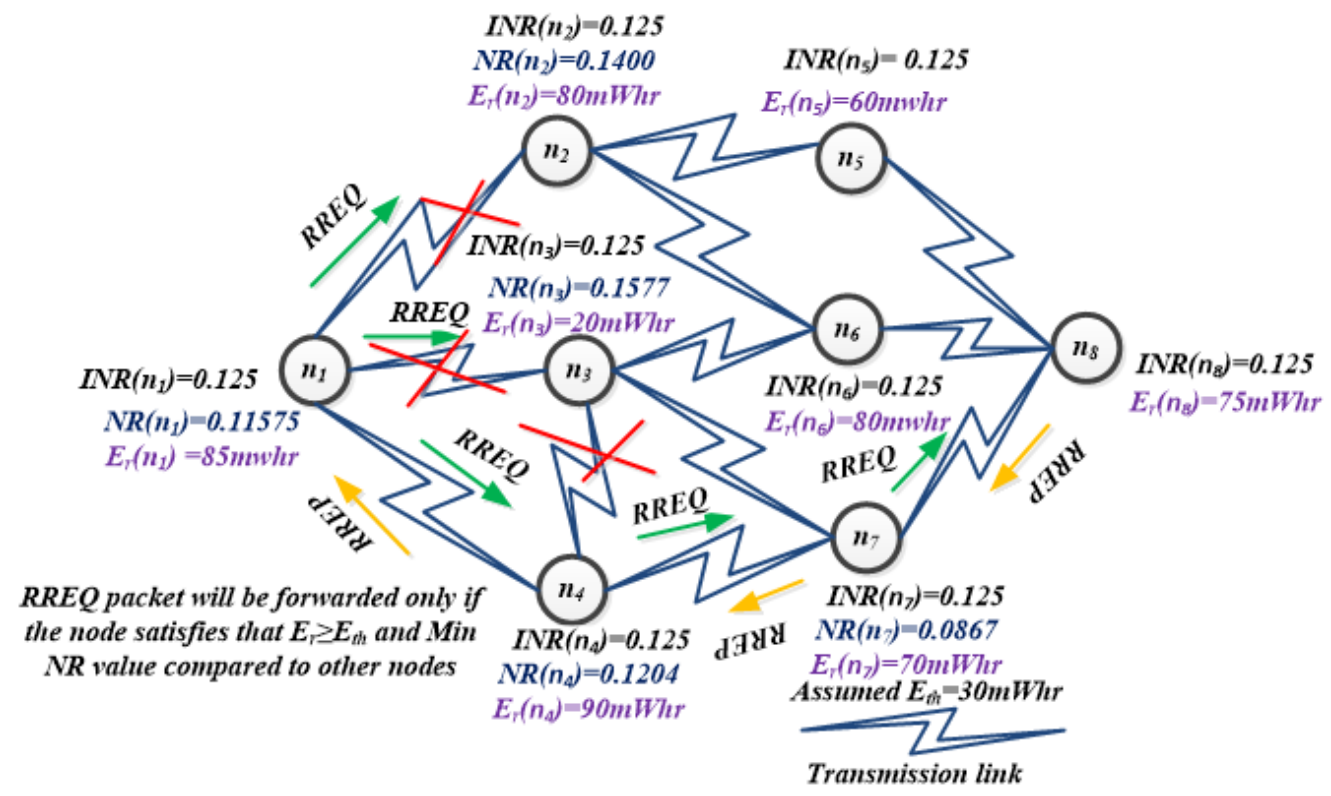

Figure 7. Energy efficient node rank based route discovery

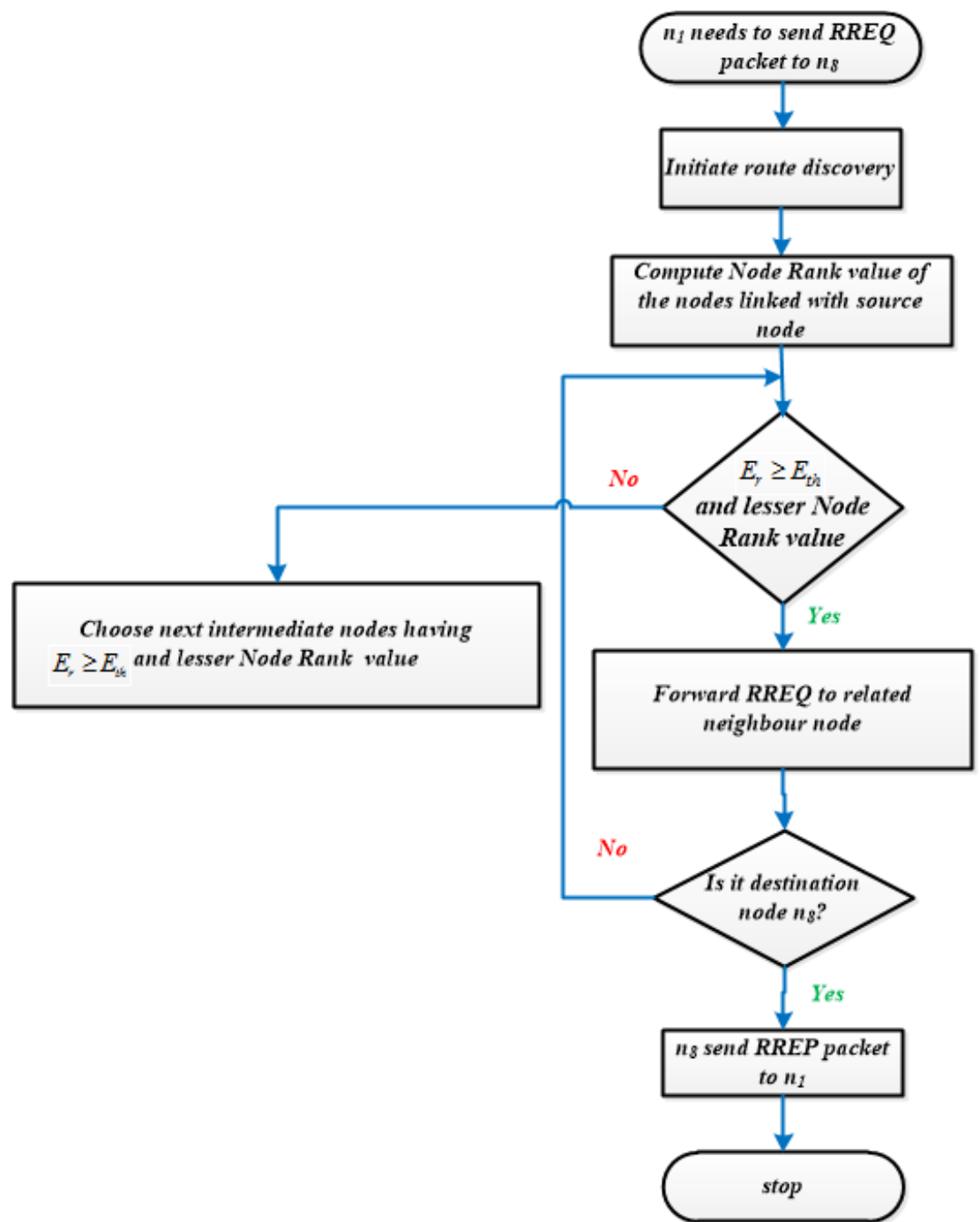

Figure 8.Flow chart representations for EENRR algorithm 


\section{RESUlTS AND DiscuSSION}

In this EENRR algorithm all works were carried out using GloMoSIM-2.03. The proposed work has experimented with various Ballistic Mobility models such as Uniform and Random Way Point mobility models [28]. As Uniform mobility model is simple, compared to Random Way Point mobility model, the proposed work is experimented only with Random Way Point mobility model. Also, the work assumes that the node's arrival follows the exponential distribution. The various parameters that were estimated during the simulation are as follows:

Packet Delivery Ratio (PDR): The ratio of the number of data packets received to that of the number of packets sent as in equation 12 .

$$
\text { PDR }=\frac{\text { Total number of data packet received }}{\text { Total number of data packet sent }}
$$

Control overhead: The sum of the number of RREQ, RREP and RERR as in equation 13.

$$
\text { Control Overhead }=R R E Q+R R E P+R E R R \text { in packets }
$$

Average Residual Energy: It is taken as the average of the residual energy levels of all the nodes in the network as in equation 14.

$$
\text { Average Residual Energy }=\sum \frac{\text { Remaining energy of individual nodes }}{\text { Total number of nodes }}
$$

These metrics were estimated by varying the following parameters:

1. Number of nodes

2. Speed $(\mathrm{m} / \mathrm{s})$

3. Number of traffic sources

\subsection{Experimental Setup}

The performance metrics like packet delivery ratios, control overhead and average residual energy were measured by varying the speed $(\mathrm{m} / \mathrm{s})$, number of source destination pairs, number of nodes, pause time, terrain dimension parameters etc., in the configuration file are shown in the Table 1.

Table 1.Parameters used for simulation

\begin{tabular}{|l|l|}
\hline Parameters & Value \\
\hline Simulation Time & $15 \mathrm{~m}$ \\
Terrain Dimension & $1000 \mathrm{~m} \times 1000 \mathrm{~m}$ \\
Number of Nodes & $10-100$ \\
Mobility-WP-Pause $(\mathrm{sec})$ & $10-50$ \\
Mobility-WP-Max-Speed $(\mathrm{m} / \mathrm{s})$ & $2-10$ \\
\hline Seed & 1 \\
\hline Node Placement & Uniform \\
\hline Mobility Model & Random-Waypoint \\
\hline Mobility WP-Min-Speed & 0 \\
\hline Mobility Position Granularity & 0.5 \\
\hline
\end{tabular}


International Journal of Computer Networks \& Communications (IJCNC) Vol.11, No.1, January 2019

\begin{tabular}{|l|l|}
\hline Propagation Limit & -111.0 \\
\hline Propagation Pathloss & Free-space \\
\hline Noise Figure & 10.0 \\
\hline Radio Type & Radio Accnoise \\
\hline Radio Initial Power Level & 4000 \\
\hline Radio Frequency & $2.4 \mathrm{e} 9$ \\
\hline Radio Bandwidth & 2000000 \\
\hline Radio Rx Type & SNR Bounded \\
\hline $\begin{array}{l}\text { Radio Rx SNR } \\
\text { Threshold }\end{array}$ & 10 \\
\hline Radio Tx-Power & 15 \\
\hline Radio-Antenna Gain & 0.0 \\
\hline Radio Rx Sensitivity & -81.0 \\
\hline Radio Rx Threshold & -81.0 \\
\hline Mac Protocol & IEEE-802.11 \\
\hline Promiscuous Mode & Yes \\
\hline Network Protocol & IP \\
\hline Routing Protocol & DSR, EEDTR \\
\hline Network Output Queue Size Per & 100 \\
Priority & \\
\hline
\end{tabular}

\subsection{Comparison of Performance with Respect to Density of Nodes}

The density of the network is varied by varying the number of nodes in the network. The node density is varied from 10 to 100 with the following constant parameters in the simulation. The speed of the node is $5 \mathrm{~m} / \mathrm{s}$, pause time is the $50 \mathrm{~s}$ and the terrain dimension is $1000 \mathrm{~m} \times 1000 \mathrm{~m}$.

Figure 9 shows the graph between the number of nodes and the average residual energy in $\mathrm{mWhr}$. When the density of the node increases from 10 to 100 , the average remaining energy of the nodes in the network is also increased as more number of intermediate nodes is required to transmit the packets in the network. The proposed EENRR algorithm increases the average residual energy by $31.08 \%$ over the existing DSR protocol and $21.26 \%$ over the existing EEDTR protocol. The proposed EENRR technique chooses lower traffic route, thus it minimizes the control packets considerably over the other protocols. Hence it minimizes the energy consumption and increases the average residual energy of the nodes in the network over the other existing protocols.

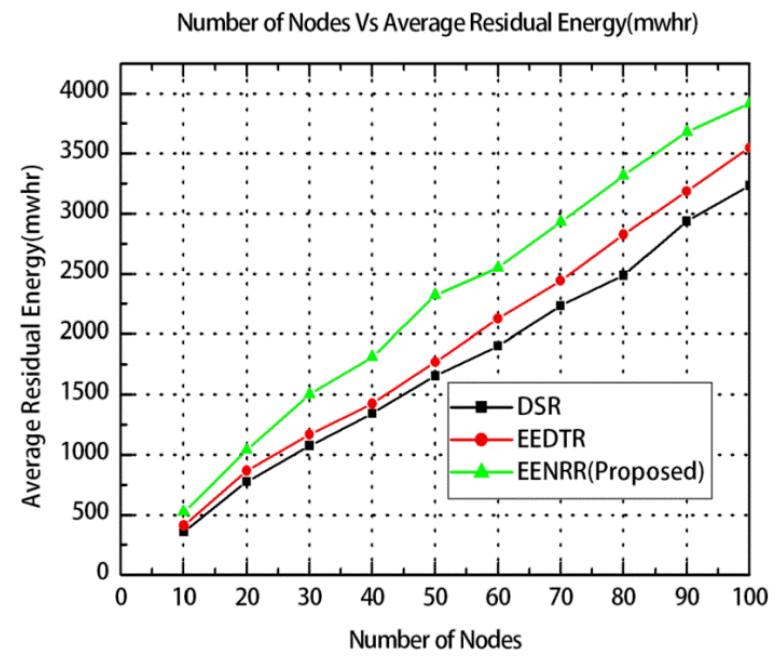

Figure 9. Number of nodes Vs Average Residual Energy (mwhr) 
Figure 10 compares the Packet Delivery Ratio of the proposed EENRR algorithm and the existing DSR and EEDTR protocols by varying the number of nodes. The simulation results show the proposed EENRR has increased the PDR value to $45.38 \%$ but it is only $28.3 \%$ for the existing DSR and EEDTR protocols. This improvement is achieved due to the selection of stable and optimal route by choosing less node rank which minimizes the traffic in the related route. Hence higher PDR is achieved.

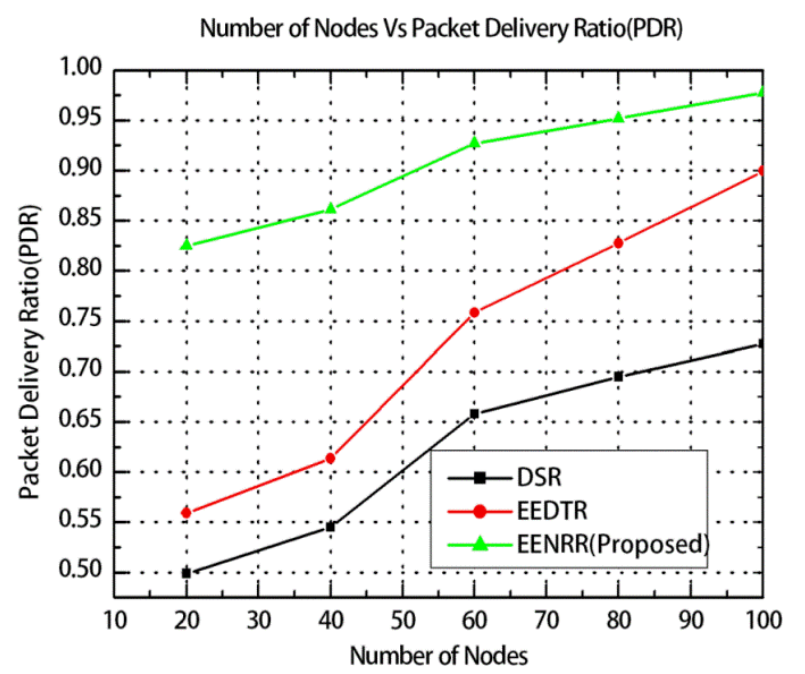

Figure 10. Number of nodes Vs Packet Delivery Ratio (PDR)

Figure 11 shows the graph between the varying number of nodes and the control overhead in packets for the proposed EENRR algorithm and compares them with the existing DSR and EEDTR protocols. When the density of the node increases from 10 to 100 , the average control overhead increases since more number of control packets are used for route discovery and maintenance. The EENRR protocol shows better performance like reduction of the control overhead by $41.15 \%$ over the DSR and EEDTR protocol by $31.51 \%$. This reduction in control overhead is achieved by minimizing the number of retransmission and route discovery by forming a more stable energy efficient route. In EENRR, the control packets like RREQ and RREP are considerably reduced by instead of flooding them. EENRR chooses only one neighbor node which will forward the RREQ to its neighbor.

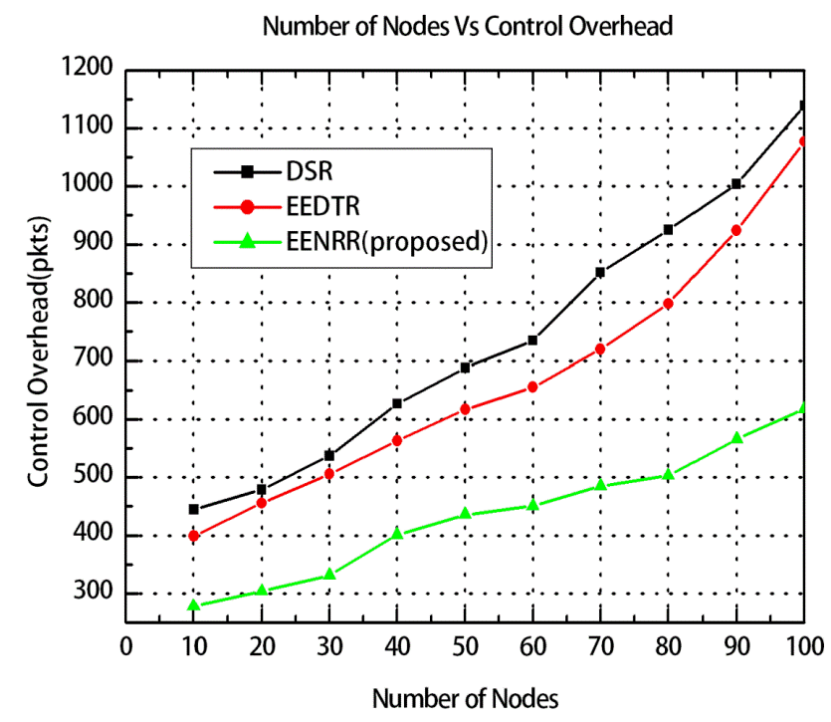

Figure 11. Number of nodes Vs Control Overhead 


\subsection{Comparison of Performance with Respect to Speed (Node Mobility)}

This section compares the performance metrics of the proposed EENRR algorithm with that of existing DSR and EENRR algorithm by varying the speed of the mobile nodes from 2 to $10 \mathrm{~m} / \mathrm{s}$ with the following constant parameters in the simulation. The network has 100 mobile nodes, the pause time of the node is 50 seconds, the number of source destination pairs (traffic sources) is 10 and the terrain dimension is $1000 \mathrm{~m} \times 1000 \mathrm{~m}$.

Figure 12 shows the graph between the varying speed of nodes in $\mathrm{m} / \mathrm{s}$ and the average residual energy in $\mathrm{mWhr}$ for the proposed EENRR algorithm with the existing DSR and EEDTR protocol. When the speed increases from 2 to $10 \mathrm{~m} / \mathrm{s}$, the average residual energy of the nodes decreases because the frequent topology change causes more overhead in route discovery which consumes more energy of the mobile nodes. The proposed protocol increases the network lifetime by increasing the average residual energy by $31.16 \%$ over the DSR protocol and $17.25 \%$ over the EEDTR protocol. As the proposed protocol chooses the minimum number of neighbors to forward the packets, the link stability will be improved and energy drain rate is also minimized.

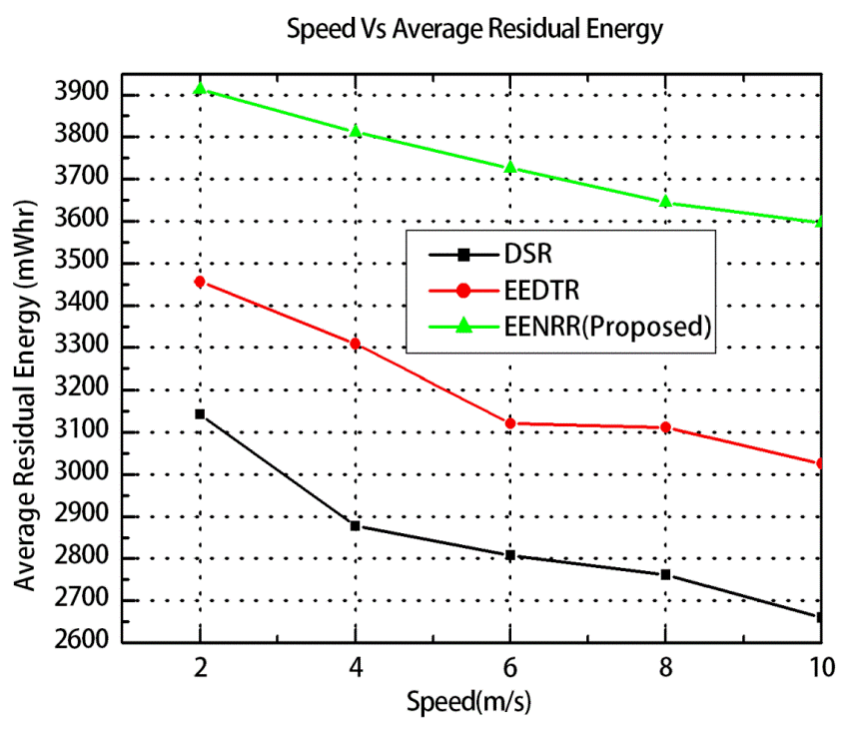

Figure 12. Speed Vs Average residual energy

Figure 13 shows the graph between varying speed of the node in $\mathrm{m} / \mathrm{s}$ and the packet delivery ratio for the proposed EENRR algorithm with existing DSR and EEDTR protocols. When the speed of the node increases from 2 to $10 \mathrm{~m} / \mathrm{s}$, the average packet delivery ratio decreases because the frequent topology change causes drop in packet delivery. The graph shows that the proposed EENRR algorithm increases the packet delivery ratio by $38.36 \%$ over the DSR protocol and $23.78 \%$ over the existing EEDTR protocol. 


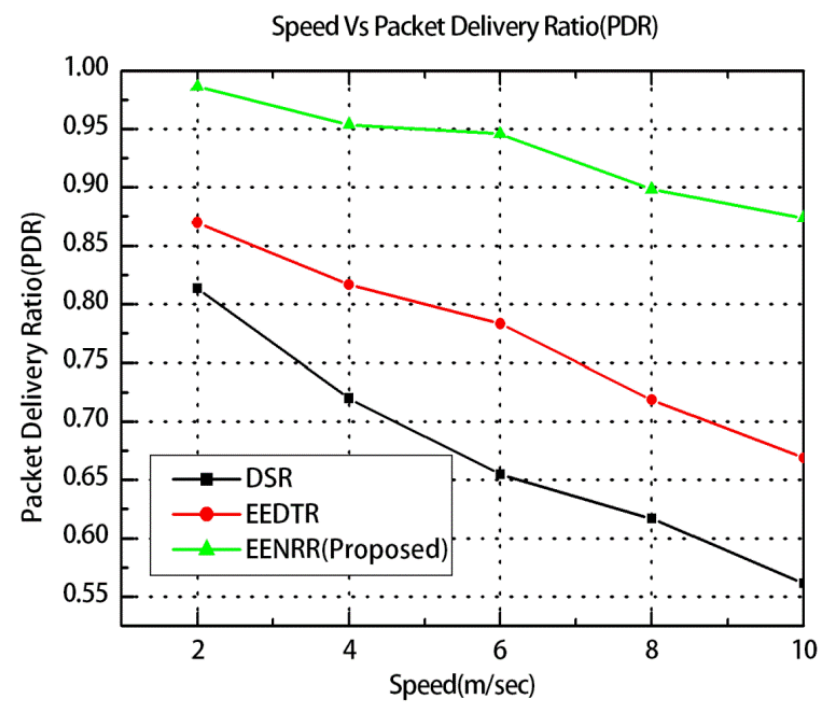

Figure 13. Speed Vs Packet Delivery Ratio

\subsection{Comparison of Performance with Respect to the Number of Source Destination Pairs (traffic sources)}

The density of the traffic sources or the number of source destination pairs is varied in the network from 5 to 30 pairs with the following constant parameters in the simulation. The node density is 100 mobile nodes, speed of the node is $5 \mathrm{~m} / \mathrm{s}$, pause time is 50 seconds and the terrain dimension is $1000 \mathrm{~m} \times 1000 \mathrm{~m}$.

Figure 14 shows the graph between varying number of source destination traffic and the packet delivery ratio for the proposed EENRR and the existing DSR and EEDTR protocols. When the traffic sources increase from 5 to 30, the average packet delivery ratio decreases because the number of routes between the source and the destination is reduced due to the minimal number of available intermediate nodes. The statistics shows that the proposed EENRR algorithm increases the packet delivery ratio over the existing DSR by $31.48 \%$ and EEDTR protocols by $7.75 \%$.

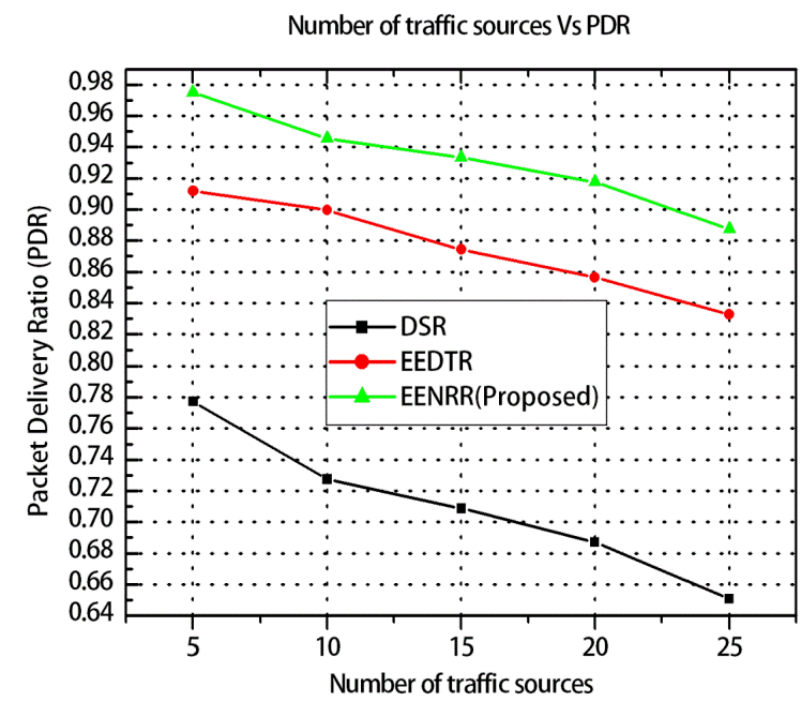

Figure 14. Number of traffic sources Vs Packet Delivery Ratio 


\section{Conclusion}

The proposed Energy Efficient Node Rank Routing (EENRR) algorithm is energy efficient with stable route. It increases the network lifetime and PDR, it also minimizes the control overhead. As this proposed EENRR forwards the RREQ packet within the neighbor nodes based on the better residual energy which is done by comparing with threshold energy and the lesser Node Rank value. It considerably reduces the flooding of the control packets and link failures. Hence the proposed EENRR protocol maximizes the network lifetime and PDR. When the number of nodes increases from 10 to 100 nodes, the proposed EENRR algorithm increases the average residual energy by $31.08 \%$ over the existing DSR protocol and $21.26 \%$ over the existing EEDTR protocol. Similarly, it increases the PDR by $45.38 \%$ and $28.3 \%$ over the existing DSR and EEDTR protocols respectively. The drawback of this proposed algorithm is, it could forward the packets within the limited range as well as act as a temporary network. In the future, works can be focussed to increase the number of nodes as well as coverage area to evaluate the performance routing.

\section{ACKNOWLEDGEMENTS}

The authors thank S R Engineering College (Autonomous) management for providing the facility regarding research works.

\section{REFERENCES}

[1] C. Daniel Nesa Kumar \&V. Saravanan,(2018)“A Survival Study on Energy Efficient and Secured Routing in Mobile Ad-hoc Network", IOSR Journal of Computer Engineering, Vol. 20, Issue No. 1, pp. 35-46.

[2] M.N. Kalpana, K.N. Sridevi \& Jitendranath Mungara, (2013)“Enhanced Energy Efficient Routing for Self Adaptive Geographic Routing In MANETs", International Journal of Computational Engineering Research, Vol. 03, Issue. 7,

[3] Senthil Murugan Tamilarasan \&Kannan Eswariah,(2013) "Link Stability With Energy Aware Ad Hoc On Demand Multipath Routing Protocol In Mobile Ad Hoc Networks", American Journal of Applied Sciences, Vol.10, No. 8, pp. 844-848.

[4] D. Kothandaraman, A. Amuthan, C. Chellappan \&N. Sreenath,(2011) "Prevention of Vulnerabilities on Location- based Geocasting and Forwarding Routing Protocol in Mobile Ad-hoc Network", International Journal of Security and Its Applications Vol. 5, No. 1, pp.65-76

[5] C. Siva Ram Murthy \&B.S. Manoj,(2007),"Ad hoc Wireless Networks Architecture and Protocols”, Pearson Education Publication, 2006, pp. 1- 878.

[6] Rashmi Rohankar, Rinkoo Bhatia, Vineet Shrivastava \& Deepak Kumar Sharma, (2012),"Performance Analysis of Various Routing Protocols (Proactive and Reactive) for Random Mobility Models of Ad-hoc Networks", 1st Int. Conf. on Recent Advances in Information Technology , RAITDhanbad, India , 15-17 March.

[7] S. Singh \&C. Raghavendra, (1998) "PAMAS: Power Aware Multi-Access protocol with Signalling for Ad hoc Networks", ACM Computer Communication Review, Vol. 28, No. 3, pp. 5-26.

[8] Baisakh, (2013)“A Review of Energy Efficient Dynamic Source Routing Protocol for Mobile Ad Hoc Networks", International Journal of Computer Applications, Vol. 68, No. 20, pp. 0975 - 8887 
[9] Z. Zhang, G. Mao \&B.D.O. Anderson, (2015)“Energy-Efficient Broadcast in Mobile Networks Subject to Channel Randomness, Wireless Communications", IEEE Transactions, Vol.14, No.6, pp. 2929-2941.

[10] Rafael Pérez TorresCésar, Torres-HuitzilHiram \&Galeana-Zapién,(2016)"Power management techniques in smartphone-based mobility sensing systems: A survey", Pervasive and Mobile Computing, Vol. 6, pp. 1-21.

[11] Ming Shen Jian \&Chienting Lin., (2005) "Improved rank-based multi-path routing in mobile ad-hoc networks", The 7th International Conference on Advanced Communication Technology, ICACT.

[12] Deepti Badal, Gwalior \&Rajendra Singh Kushwah, (2016)“Energy Efficient Approach of Route Selection for Dynamic Source Routing Protocol in MANET", ProceedingAICTC '16 Proceedings of the International Conference on Advances in Information Communication Technology \& Computing,Bikaner, India.

[13] Rjab Hajlaoui, Sami Touil \&Wissem achour, (2015)“O-DSR: Optimized DSR Routing Protocol for Mobile Ad Hoc Network”, International Journal of Wireless \& Mobile Networks, Vol. 7, No. 4. pp. 37-47

[14] Sukhdev Singh Ghuman,(2016)“Dynamic Source Routing (DSR) Protocol in Wireless Networks", IJCSMC, Vol. 5, Issue. 6, pp. 251-254

[15] Navin Mani Upadhyay \&Kunal Gaurav Arun Kumar,(2014) "Modified DSR, an energy conserving approach to DSR protocol in MANET", Published in: 2014 International Conference on Communication and Signal Processing,pp. $109-112$

[16] M. Tamilarasi, S. Chandramithi \&T. G. Palanivelu,(2008) “Overhead Reduction and Energy Management in DSR for MANET”, Proc. Of COMSWARE,), pp. 762-766.

[17] R. Dube, Rais, C. D. Kuang Yeh Wang \&S. K. Tripathi,(1997)“Signal Stability based Adaptive Routing (SSA) for Ad-Hoc Mobile Networks,” IEEE Personal Communications, Vol. 4, Issue. No. 1, pp. 36-45.

[18] E. M. Royer \&C. K. Toh, (1999)“A Review of Current Routing Protocols for Ad Hoc Mobile Wireless Networks”, IEEE Personal Communications, Vol. 6, Issue. No. 2, pp. 46-55.

[19] S. Chakrabarti \&A. Mishra, (2001)“QoS Issues in Ad Hoc Wireless Networks”, IEEE Communications Magazine, Vol. 39, No. 2,

[20] Hui Xia, Jia Yu, Zhen-Kuan Pan, Xiang-Guo Cheng \&Edwin H. Sha., (2016)“Applying trust enhancements to reactive routing protocols in mobile ad hoc networks", Journal Wireless Networks archive, Vol. 22, Issue No. 7, pp. 2239-2257

[21] S. Sridhar \&R. Baskaran,(2015) "Efficient Routing in Mobile Adhoc Networks Emphasizing Quality of Service by Trust \& Energy based AODV”, Journal of Communications Software and Systems, Vol. 11, No. 1, pp.1-7

[22] C.K. Toh, (2001)"Maximum Battery Life Routing to Support Ubiquitous Mobile Computing in Wireless Ad Hoc Networks”, IEEE Communications Magazine, Vol. 39, Issue No. 6,

[23] Shuchita Upadhayaya \&CharuGandhi,(2010)"Node Disjoint Multipath Routing Considering Link and Node Stability protocol:Acharacteristic Evaluation", International Journal of Computer Science, Vol.7, Issue 1, No.2, pp. 18-25

[24] P. Sivasanka, C. Chellappan \&S. Balaji,(2011) "Energy Efficient Routing Algorithms and performance comparison for MANET", CIIT International Journal of Wireless Communication, Vol. 3, No.10. 
[25] M. Venkata Ramana \&P. Venkata Krishna, (2016) “An approach for routing in delay tolerant mobile ad-hoc networks using quadrant-based look-ahead method", International Journal of Wireless and Mobile Computing, Vol. 10, Issue 3,

[26] Bhed B. Bista \&Danda B., Rawat, (2017) "EA-Epidemic: An Energy Aware Epidemic-Based Routing Protocol for Delay Tolerant Networks”, Journal of Communications, Vol. 12, No. 6, pp. 304-311

[27] D. Jyothi Preshiya \& C.D. Suriyakala, (2015)“A Survey of Factors Influencing Network Lifetime in Delay Tolerant Network”, Indian Journal of Science and Technology, Vol 8, No. 15, pp. 1-9

[28] Federica Bogo \&Enoch Peserico, (2013)“'Optimal Throughput and Delay in Delay-tolerant Networks with Ballistic Mobility”, MobiCom 13 Proceedings of the 19th Annual International Conference on Mobile Computing \& Networking, pp.303-314.

[29] S Aluvala, D Vodnala, N Yamsani, S Phani Kumar, (2014) "A routing algorithm for localization of link failure in MANET", International Journal of Current Engineering and Scientific Research(IJCESR), Vol.1, Issue. 3, pp. 25-30.

\section{Authors}

Dr. D. Kothandaraman received his B.E. computer Science and Engineering from Dr. Pauls Engineering College (Anna University), M.Tech., in Information Security from Pondicherry Engineering College(Pondicherry University(Govt. of India)) and Ph.D. in Computer Science and Engineering from College of Engineering, Guindy, Anna University(Govt. of Tamil Nadu). To his credit, he has 7 years of teaching and research experience. His area of research interest is computer networks, Wireless Sensor Networks (WSN), Mobile Ad-hoc Networks (MANETs) and Internet of Things (IoT). He has published various papers in International Journals and in conferences.

Dr. C. Chellappan is a Professor (retd.) in the Department of Computer Science and Engineering at Anna University, Chennai, India. He received his B.Sc. in Applied Sciences and M.Sc in Applied Science- Applied Mathematics from PSG College Technology, Coimbatore under University of Madras. He received his M.E and Ph.D in Computer Science and Engineering from Anna University. He held in various positions during his working periods. He has published more than 200 papers in reputed International Journals and Conferences. His research areas are Image Processing, Computer Networks, Cyber security Distributed Computing, Network Security, Mobile

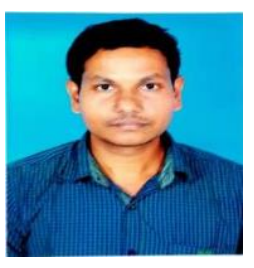

Agents. Currently working as the senior Professor and Principal in G.K.M. College of Engineering, Perungalathur, Chennai.

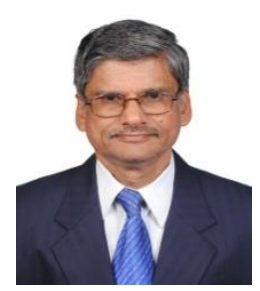

